\title{
Identidad local y ejercicio de la ciudadanía en jóvenes de Villa El Salvador
}

\section{Local identity and citizenship in young people Villa El Salvador}

Daysi Karol Pacheco Sánchez

https://orcid.org/0000-0003-4918-8414

Universidad Nacional Federico Villarreal, Perú

Segundo Waldemar Rios Rios

https://orcid.org/0000-0003-1202-5523

Universidad Norbert Wiener, Perú

Autor para correspondencia: waldoigv@hotmail.com; karol.pacheco.sanchez@gmail.com Fecha de recepción: 20 de julio del 2019 - Fecha de aceptación: 15 de diciembre del 2019

\section{Resumen}

La presente investigación tuvo como objetivo principal establecer la relación entre identidad local y el ejercicio de la ciudadanía en un grupo de 195 jóvenes (50.4\% mujeres) de Villa El Salvador entre 14 y 29 años, a través de un estudio correlacional, con el fin de describir y analizar la relación entre ambas variables de estudio. Para la evaluación de estas variables se elaboraron dos escalas: La Escala de Identidad Local (ESIL) y la Escala del Ejercicio de la Ciudadanía (EECI), de las cuales se reportaron evidencias de validez basadas en el contenido, el constructo y confiabilidad por consistencia interna, obteniéndose resultados satisfactorios para ambas escalas. Asimismo, se encontraron niveles promedio de identidad local y ejercicio de la ciudadanía en la muestra de jóvenes de Villa El Salvador. Por otro lado, al establecer la relación entre la identidad local y el ejercicio de la ciudadanía, se encontró una correlación de Pearson $(r=.653, p<.01)$, esto nos indica que existe una relación positiva altamente significativa entre las variables de estudio. Estos hallazgos sugieren que cuanto mayor es el nivel de identidad local mayor es el nivel del ejercicio de la ciudadanía.

Palabras Clave: identidad local; identidad; ejercicio de la ciudadanía; participación; ciudadanía

\begin{abstract}
This research's main objective was to establish the relationship between local identity and citizenship in a group of 195 young people from Villa El Salvador between 14 and 29, through a correlational study, in order to describe and analyze the relationship between the two variables of study. For the evaluation of these study variables were developed, the Local Identity and scale ESIL Exercise of Citizenship EECI scale, which have a high level of validity and reliability with Cronbach's alpha coefficient of 0.86 respectively for each. Also an average local identity with an average of 60.71, in the sample of young people from Villa El Salvador, on the level of citizenship was an average level according to the average of 72.74 was found. Moreover the main objective of the study was to establish the relationship between local identity and citizenship, finding a correlation of Pearson $(r=0.624, p<0.01)$, this indicates that there is a positive highly significant
\end{abstract}


relationship between the variables study. This means that the higher the level of identity is more local level citizenship.

Key words: local identity; identity; citizenship; participation; citizenship

\section{Introducción}

La identidad local ha sido caracterizada con una serie de teorías que tratan de explicar por qué adquirimos percepciones particulares y conductas, con respecto del entorno geográfico y cultural al que pertenecemos y nos diferencian de otras esferas culturales, en las que interactuamos. Algunos autores señalan que la identidad es una construcción social en evolución, que se va transformando, enriqueciendo, renovando, sin que esto implique descalificación o pérdida de los parámetros anteriores. Nidding (2001).

Sobre los estudios sobre el ejercicio de la ciudadanía, autores como Sermeño (2004) explican que la noción de ciudadanía tiene tres componentes: la posesión de un conjunto de derechos y deberes; la pertenencia a una comunidad política determinada (Estado-nación); y la capacidad de participar en la definición de la vida pública (política social y cultural). Jurídicamente la ciudadanía está definida por un conjunto de derechos y deberes obligatorios para los individuos que comparten un territorio denominado nación.

Es importante señalar que existen pocos estudios acerca de la relación entre identidad local y ejercicio de la ciudadanía de ahí que cobra mucho valor esta investigación porque ha aportado bases metodológicas a través del diseño de dos escalas, la primera para medir la identidad local y la segunda para medir el ejercicio de la ciudadanía en jóvenes respectivamente, lo cual es un legado que permitirá obtener información confiable para futuras investigaciones.

En este artículo se aborda en el primer capítulo el planteamiento del problema de investigación, en el segundo capítulo se revisa las investigaciones sobre las teorías desarrolladas en identidad local y en ejercicio de la ciudadanía, en seguida desarrollamos el marco conceptual que estuvo enmarcado a definir las variables de estudio y los campos de acción en el tercer capítulo está referido a la metodología aplicada en la investigación donde se presenta la descripción del diseño de investigación utilizado, las características sociodemográficas de la población de estudio, la forma como se ha elegido el tamaño y la selección de sujetos para la muestra. A su vez, se describen los instrumentos utilizados y finalmente los procedimientos que detallan el modo en que se llevó a cabo el estudio. En el cuarto capítulo se presentación los resultados de acuerdo a los análisis estadísticos.

Finalmente, se discuten los resultados encontrados, comparando y contrastando los hallazgos con algunos estudios similares. También, se formulan las conclusiones y se presentan algunas sugerencias para futuras investigaciones, que permiten ampliar el campo de investigación propuesto, con la finalidad de implementar programas de intervención.

\section{Problemática}

La investigación se realizó en el distrito de Villa El Salvador, donde en los últimos años han desarrollado experiencias exitosas para fomentar el ejercicio de la ciudadanía en los jóvenes, 
se puede destacar la implementación de espacios de participación, programas municipales y espacios juveniles, estas acciones suman a los esfuerzos por la ejecución del Plan Integral de Desarrollo Concertado de Villa El Salvador al 2021 - PIDCVES 2021, a la línea estratégica No 06: Democracia participativa y ciudadanía (Municipalidad de Villa El Salvador, 2006).

Lo pobladores de Villa El Salvador tienen características peculiares que los distinguen con pobladores de otros distritos, la historia del desarrollo autogestionario de Villa El Salvador, fue el primer experimento económico y social que envolvió a un sector de pueblos jóvenes al sur de los distritos tradicionales de Lima Metropolitana que se organizó basado en una idea conceptualizada bajo la modalidad de cooperativas o asociaciones. Las identidades, particularidades y pluralidades locales tienen garantía constitucional, y deben ser respetadas pues ellas son cimiento de nuestra identidad nacional (Dromi, 2007).

Todo individuo que se halla dentro de un entorno local, tiene un determinado nivel de ciudadanía dentro de su entorno social, así también de manera inherente se cuenta con un grado de identidad local. (Pacheco, 2010).

El estudio relaciona el concepto de ejercicio de la ciudadanía fundamentalmente con la participación que tienen los sujetos en diversas actividades, grupos y procesos sociales, tales como la participación en actividades formales, en organizaciones escolares, en el acceso a información política a través de los medios de comunicación, entre otros. Estos elementos se relacionan con diversos aspectos y procesos de integración social y, por lo tanto, son centrales en el aprendizaje de los estudiantes acerca de quiénes son y cómo se viven la democracia y la ciudadanía (Municipalidad de Villa El Salvador, 2006).

$\mathrm{Al}$ estar la identidad relacionada con la vida del hombre, la misma estará determinada por su circunstancia, por su lugar, por su historia, y así como existe una identidad personal, existe una identidad colectiva, existe una identidad local, común a los vecinos de determinado lugar y diferente a las de otros lugares. Es así que la participación juvenil se enlaza con la identidad local que tiene ver con lo que somos, con nuestra historia de vida. Es aquello que nos caracteriza y distingue de los demás; es una necesidad afectiva y una realidad concreta que tiene que ver con el identificarse y con reconocerse y que presupone la existencia de otro del cual distinguirse y diferenciarse (Dromi, 2007). Rostworoski (1987) en su artículo Somos un país acomplejado, presenta un breve comentario en el cual destaca que no somos nación dado que no tenemos el sentimiento de unidad, de ser uno. Señala la autora que el peruano es un ser acomplejado porque ha pasado por una serie de fracasos que se dan desde la conquista por los españoles, la presencia de hombres venidos de otros lugares para liberarnos y la perdida de la guerra con Chile; factores que han mermado la formación de la conciencia nacional. Finalmente, todo individuo que se halla dentro de un entorno local, tiene un determinado nivel de ciudadanía dentro de su entorno social, así también de manera inherente se cuenta con un grado de identidad local. (Pacheco, 2010). Motivados por estas influencias se tuvo como objetivo general relacionar la identidad local y el ejercicio de la ciudadanía en un grupo de jóvenes de Villa El Salvador. Los objetivos específicos fueron estimar evidencias de validez y confiabilidad de los instrumentos elaborados para medir las variables de estudio y relacionar la identidad local y el ejercicio de la ciudadanía en un grupo de jóvenes de Villa El Salvador, según las variables de control (sexo, grado académico y edad). 


\section{Marco teórico}

En este apartado se recoge los aportes de autores relevantes sobre las dos variables de nuestra investigación como son: la identidad local y sobre el ejercicio de la ciudadanía.

\section{Identidad local como construcción social}

Según el diccionario de la Real Academia de la Lengua Española (2013) identidad es el conjunto de rasgos propios de un individuo o de una colectividad que los caracterizan frente a los demás; conciencia que una persona tiene de ser ella misma y distinta a las demás.

Es decir, se plantea a la identidad como aquel conjunto de procesos sociales de significación, o de un modo más complejo, del conjunto de procesos sociales de producción, circulación y consumo de la significación en la vida social. (García Canclini, 1999). En este sentido, el autor confirma la idea de que la identidad se construye a través de las historias de vida de los individuos, se mantiene en la memoria, se transforma a lo largo de las experiencias personales $-\mathrm{y}$ colectivas- y se releja en los diferentes aspectos sociales según sea el caso. (De Zan, 2008).

Con relación a lo anterior y con el objetivo de complementar la idea de construcción de identidad desde el individuo y proyectarlo hacia lo social, Giménez (2007) también distingue los atributos de pertenencia social y particularizantes. Donde los primeros se conforman de manera colectiva y los segundos de manera individual, pero -y es importante esta acotación-, los atributos particularizantes son una constante construcción del ciudadano tanto a su interior como al exterior ayudado por el colectivo. Es decir, hay una relación dialéctica en la construcción de identidad del individuo a nivel personal, se reconoce en el colectivo, a su vez alimenta al colectivo y se construye a partir de él (Cruz y Pedraza, 2011).

Entonces se puede concluir que la identidad de una ciudad, de una región, es la personalidad construida por su comunidad. Lo que ella es y pretende ser, no por su materialidad, sino por su espíritu. Es su ser histórico, filosófico, ético y de comportamiento. Es lo que la hace individual, singular y la distingue del resto. Es un conjunto de atributos o características con los que la comunidad se identifica y con los cuales quiere ser identificada por los visitantes. Capriotti (1992).

La construcción de la identidad se hace a partir de materiales de la "historia, geografía, biología, instituciones productivas y reproductivas, memoria colectiva y fantasías personales, aparatos de poder y revelaciones religiosas" (Castells, 1997, p.29) en un todo que es la cultura. La identidad es, por tanto, un constructo cultural que se aloja en la subjetividad de los ciudadanos y que les permite una clasificación, es decir, diferenciar a los "propios" de los "otros". Es al mismo tiempo, real y objetiva, subjetiva e imaginada, construida y dada, adquirida (cultura) y dada (natural). Es la imagen de lo que somos frente a los demás. 
En síntesis, como plantea Nidding (2001), la identidad es una construcción social que se va enriqueciendo, renovando, en definitiva transformado, sin que ello necesariamente implique desvalorización o pérdida de los parámetros anteriores.

La identidad está ligada a la historia y al patrimonio cultural y en este sentido se dice que no existe sin la memoria, sin la capacidad de reconocer el pasado, sin elementos simbólicos o referentes que le son propios y que ayudan a construir el futuro. Por eso se reconoce como un concepto dinámico que se recrea individual y colectivamente y se alimenta continuamente de la influencia exterior (Zúñiga y Asun, 2003).

Heller (2002) en su -tal vez obra más importante-Sociología de la vida cotidiana y Rizo (2005), mencionan la asociación de individuos como parte fundamental en la búsqueda de reconocimiento y formación de identidad.

La identidad regional se refiere al proceso a través del cual las personas identifican un territorio, con su cultura, tradiciones, paisaje, historia. Zúñiga y Asun (2003). La definen como "aquella parte del autoconcepto de un individuo basada en su pertenencia a un grupo regional, junto con el significado valorativo y emocional asociado a dicha pertenencia” (p. 75), lo que se puede manifestar de diversas formas, como complejo de inferioridad o como orgullo, como sentimiento de pertenencia, derecho y proactividad cultural o política

La identidad regional no puede entenderse sólo en términos del pasado o presente, sino también en términos de futuro y de los proyectos, anhelos, ideales y sueños con que la gente de una región o de un país se identifica en metas, horizontes de expectativas o utopías, por definición inalcanzables en su totalidad, aunque por lo mismo fuertemente motivadoras y productivas.

En síntesis, la identidad regional implica un compromiso afectivo vital con el pasado, presente y futuro de los procesos económicos - sociales y culturales que acaecen en una localidad o región (Zúñiga y Asun, 2003).

En la definición de una identidad colectiva o social es posible distinguir en general dos tendencias. La primera, vinculada a la forma tradicional de comprender a las identidades de las sociedades, en torno a un enfoque esencialista, donde la identidad es comprendida como una "esencia manifiesta en los modos de vida, en las costumbres locales, en las instituciones establecidas, en la tradición, la cultura, y el carácter de una comunidad". La segunda rescata "el espíritu de la época", a través de las líneas generales de la historia de nuestro tiempo y lo que se considera "la dirección general y la significación de esa historia" (Geertz, 2000). En ese marco, las sociedades se entienden como «construcciones histórico - simbólicas» y, a diferencia de aquellos que enfatizan la centralidad de los lazos primordiales, serán las pertenencias y los códigos culturales, los que establecen "marcas de origen" que definen los límites entre "nosotros" y los "otros", a la vez que permiten establecer una relación particular sin por eso perder contacto con la realidad cambiante (Geertz, 2000).

Pensar la identidad en términos de construcción, nos remite a cambiar concepciones que tratan de ver a la identidad como algo "natural". Desde esta perspectiva, la identidad es 
considerada un proceso de construcción de la sociedad a la que es posible acceder a través de sus símbolos, representaciones, conductas e imágenes que los propios grupos sociales construyen a través del tiempo, "reinventando" su sentido de pertenencia en forma permanente, por lo que a diferencia de las concepciones esencialistas no tiene un final, un cierre definitivo, un modelo acabado por y para siempre.

La identidad es transformada continuamente de acuerdo a las maneras en que somos representados y tratados en los sistemas culturales que nos rodean. La identidad no es algo dado ni algo que pueda ser impuesto; se construye y se internaliza en torno de significados "con aportes de la historia, geografía, biología, instituciones productivas y reproductivas, memoria colectiva y fantasías personales" (Castells, 1997, p. 29). La historia y la memoria colectiva tienen una gran importancia en la formación de la identidad del individuo. Por memoria histórica se entiende la secuencia de eventos cuyo recuerdo conserva la historia nacional, y la memoria colectiva es una reconstrucción desordenada, donde se mezclan los recuerdos de los diversos miembros de un grupo social, cargados de afectividad, subjetividad que se confunde con el presente, mientras la historia registra y esquematiza, ordenando el pasado.

\section{La ciudadanía como concepto complejo}

Desde la década de los noventa, el concepto de ciudadanía ha vuelto a ocupar un lugar relevante en las ciencias sociales. Este renovado interés está provocado por razones de diversa índole, entre las que cabe destacar: las transformaciones en el modelo de Estado-nación; el resurgimiento y redefinición de los nacionalismos; la quiebra del consenso en torno al Estado de Bienestar y a la extensión de los derechos sociales; la intensificación de los procesos migratorios; la creciente apatía política y el deterioro de las cualidades morales de los ciudadanos, tan señalados por algunos autores; y la crítica política de los nuevos movimientos sociales (Kymlicka y Norman, 1997; Morán y Benedicto, 2000; Pérez Ledesma, 2000).

Sermeño (2004) explica que la noción de ciudadanía tiene tres componentes: la posesión de un conjunto de derechos y deberes; la pertenencia a una comunidad política determinada (Estado-nación); y la capacidad de participar en la definición de la vida pública (política social y cultural). Jurídicamente la ciudadanía está definida por un conjunto de derechos y deberes obligatorios para los individuos que comparten un territorio denominado nación.

En el plano político-ideológico la ciudadanía implica una identificación de comunidades que corresponden a la noción formal de nacionalidad. Para la sociología el concepto de ciudadanía implica relaciones de integración social, que a menudo producen fenómenos de exclusión en ámbitos comunitarios donde sus individuos aparentemente comparten condiciones de homogeneidad (Sojo, 2002).

La noción de ciudadanía es un campo de actual debate en tanto implica nociones de nosotros, ellos o los otros. Es decir, se es ciudadano ante quienes no lo son. El concepto en sí encierra la contradicción de la pertenencia a un estatus y un valor universal, Sermeño (2004) escribe que: 
...la ciudadanía es, ciertamente, un reconocido estatus de inclusión y pertenencia que apela a la existencia de una estructura de derechos universales. Pero también es un proceso histórico, resultado de una diversidad de prácticas y/o dinámicas que a su vez han seguido su propio patrón, por llamarle de alguna manera, de interpretación nacional particular. (p.88).

Si bien el concepto de ciudadanía puede ser una mirada teórica del comportamiento humano, también es un ejercicio cotidiano, una práctica social (Sojo, 2002). Asimismo, el debate acerca de la ciudadanía cobra importancia en lo que respecta a la definición de las políticas públicas de cualquier país. Dicho concepto implica un ejercicio o práctica ciudadana que involucra aspectos políticos, económicos, sociales y culturales, que van desde el sufragio para la elección de gobernantes, hasta concepciones con respecto a antagonismos de género, étnicos y ambientales.

El concepto de ciudadanía también adquiere complejidad en sus posibilidades de re significación, puesto que hoy en día en que la especie humana está auto-amenazada es difícil tener claridad de lo que puede significar la ciudadanía y las nuevas solidaridades que son necesarias para la construcción de un mundo menos desigual para la humanidad. Para lo cual la noción de ciudadanía y el ejercicio que conlleva en las esferas pública y privada deben concatenar la práctica solidaria y responsable en la dimensión local, nacional y planetaria. La comunidad de destino en el que todos estamos involucrados (Morin, 2002), implica la construcción de una identidad humana común, que podría significar la construcción de una ciudadanía planetaria, ya que es:

...la progresión y el anclaje de esta conciencia de pertenencia a nuestra patria terrestre son los que permitirán el desarrollo, por múltiples canales, en las diferentes regiones del globo, de un sentimiento de unión y de intersolidaridad necesaria para civilizar las relaciones humanas. Son el alma y el corazón de la segunda globalización, producto antagónico de la primera, los únicos que permitirán humanizar esta globalización (Morin, 2002, p.77)

Privilegiar una noción activa de la ciudadanía significa desviar el foco de atención desde los aspectos formales hacia las prácticas en que ésta se concreta, esto es, hacia los modos de participación política y ciudadana. Así, B. S. Turner concibe la ciudadanía como "el conjunto de prácticas (jurídicas, políticas, económicas y culturales) que definen a una persona como un miembro competente de su sociedad, y que son consecuencia del flujo de recursos de personas y grupos sociales en dicha sociedad" (Turner, 1993, p. 2).

Desde su teoría relacional de la sociedad, Donati entiende la ciudadanía como relación social y no como un elemento jurídico; se trata, ante todo, de una realidad socialmente producida, que tiene su historia y su evolución. Para el autor, la ciudadanía no es ya la relación que se tiene con el vértice de la sociedad, no es ya la relación política más elevada, sino que se convierte en una relación de redes, aplicable a todo el nivel del obrar social (Donati, 1999, p. 3738). Además, la ciudadanía no tiene sólo un carácter político, sino que implica también otras dimensiones de la realidad: 
...psicológicas (por ejemplo, ciertos sentimientos de pertenencia), culturales (por ejemplo, ciertos modelos de vida que vienen representados como características nacionales), económicas (el estar insertados en un cierto sistema de intercambios y de transferencia de recursos), sociales (el compartir ciertas reglas de vida), jurídicas (el referirse a cierta ordenación codificada de derechos y deberes (Donati, 1999, p. 40).

En este sentido, algunos autores plantean que la práctica de la ciudadanía requiere de una reactivación de la esfera pública, en la que los ciudadanos puedan actuar colectivamente e involucrarse en deliberaciones comunes sobre todos los asuntos que afectan a la comunidad política (Passerin d'Entrèves, 1992), así como de la ampliación de los canales comunitarios para aumentar las oportunidades de participación ciudadana (Crouch, 1999; Gadea, 2007).

\section{Metodología}

El diseño de la investigación corresponde a un diseño no experimental, ya que no hay manipulación directa de las variables, y es de tipo transeccional correlacional (Hernández et al., 2006), pues el objetivo es describir relaciones entre dos variables en un momento determinado. Complementariamente, también corresponde a un diseño instrumental porque está orientado al desarrollo de dos instrumentos de medición, específicamente a su adaptación y al estudio de sus propiedades psicométricas (Montero y León, 2002), tal como es el caso de la Escala de Identidad Local (ESIL) y la Escala de Ejercicio de la Ciudadanía (EECI).

Las variables de estudio son en primer lugar la Identidad local, que se describe como un conjunto de atributos o características con los que la comunidad se identifica dentro de su entorno local y con los cuales quiere ser identificada por el resto de localidades (Pacheco, 2010). La segunda variable de estudio es el Ejercicio de la ciudadanía que se define como: Las acciones que llevan a cabo las personas en diversos espacios de participación ciudadana donde aportan con ideas y actos, se vuelven participes de los cambios dentro de su entorno temporal, geográfico, económico y social. (Pacheco, 2010).

La población de jóvenes en el distrito de Villa El Salvador cuenta con una población de 114,334.00 jóvenes entre 14 y 29 años (49.6\% hombres y 50.4\% mujeres), los cuales representan el $29.9 \%$ de la población total del distrito (381,790.00 habitantes), según el Censo realizado por el INEI (2007). La muestra estuvo conformada por 195 jóvenes (49.7\% hombres y 50.3\% mujeres) entre 14 y 29 años, pertenecientes a nueve Instituciones Educativas, universidad y un instituto del distrito de Villa El Salvador. El muestreo que se utilizó fue no probabilístico, pues este tipo de muestreo es usado muy frecuentemente por la facilidad con que puede obtenerse una muestra; aun cuando se desconozcan las bases para su ejecución. (Sánchez \& Reyes, 2009). Así mismo es de tipo intencional porque se tuvo el acceso a los diversos espacios locales donde se encuentran los jóvenes, Instituciones educativas, el instituto y la universidad. El recojo de información se realizó en el año 2010.

Los instrumentos que se usaron para la investigación fueron: Escala de Identidad Local ESIL de Pacheco (2010), es una escala que se construyó para la investigación para lo cual se revisó la Escala de Identidad Nacional (EIN) de Portocarrero (1998), dicha escala en mención es 
un reactivo tipo Likert, cabe resaltar que la Escala de Identidad Nacional reúne, en general, adecuados criterios de validez y de confiabilidad, la escala se elaboró con el objetivo principal de conocer el nivel de identidad local en jóvenes y adolescentes. Está conformada originalmente por 35 ítems que cuentan con cinco categorías de respuesta; cada una de las cuales reciben puntuaciones de 5 a 1 según medición ordinal de escalamiento Likert. Las categorías son "Muy de Acuerdo" (5), "De Acuerdo" (4), "Indeciso" (3), "En Desacuerdo" (2) y "Muy en Desacuerdo" (1). Estas puntuaciones se invierten en el ítem negativo (ítem 34). Importante es señalar que, después de la calificación de la escala, el alto puntaje obtenido sugiere que el evaluado tiene nivel alto de identidad local mientras que los puntajes bajos lo contrario.

La Escala del Ejercicio de la Ciudadanía - EECI de Pacheco (2010) tiene por objetivo medir el nivel de ejercicio de la ciudadanía en jóvenes y adolescentes, dividida en dos dimensiones. Intención de la conducta y conducta manifiesta. La versión original elaborada cuenta con 40 ítems cuentan con cinco categorías de respuesta; cada una de las cuales reciben puntuaciones de 5 a 1 según medición ordinal de escalamiento de Likert. Las categorías son "Muy de Acuerdo" (5), "De Acuerdo" (4), "Indeciso" (3), "En Desacuerdo" (2) y "Muy en Desacuerdo" (1). Importante es señalar que, después de la calificación de la escala, el alto puntaje obtenido sugiere que el evaluado tiene fuerte ejercicio de la ciudadanía mientras que los puntajes bajos indican lo contrario.

\section{Resultados}

La descripción de resultados responde a los objetivos generales y específicos del estudio, en primer lugar, se describen los resultados del análisis de las propiedades psicométricas de los instrumentos que incluye validez de contenido, confiabilidad y puntajes directos, para posteriormente, describir los resultados de la aplicación de los cuestionarios (ESIL y EECI) y exponer la relación existente entre las distintas subescalas que componen ambos cuestionarios.

\section{Análisis psicométrico de la Escala de Identidad Local ESIL. (Pacheco, 2010).}

Sobre las propiedades psicométricas de la Escala de Identidad Local ESIL, los resultados de la validez de contenido indican que dieciséis (16) de los treinta cinco (35) ítems originales presentados a los jueces fueron aceptados como representativos del universo de contenido de la Escala de Identidad Local, pues los coeficientes $V$ de Aiken fueron significativos a una $p<.05$, que en el caso de 10 jueces deben ser mayores a .80 (Escurra, 1989), lo que garantiza que la prueba presenta validez de contenido.

Al establecer la validez de los ítems de la Escala de Identidad Local ESIL, a través del criterio de constructo mediante las correlaciones ítem-test se apreció que 15 ítems de los 16 aceptados por el criterio de jueces presentaron correlaciones significativas mayores a .30 .

El Análisis Factorial Exploratorio AFE, exploró si los datos mostraban bondad de ajuste respecto a la matriz de relaciones lineales, condición fundamental para realizar el análisis factorial. Se obtuvo una matriz de correlaciones diferente de cero, Barttlett- $\chi 2(\mathrm{gl}: 105)=923.9$, $\mathrm{p}<.01$; KMO de 0.87, valor considerado adecuado (Hair, Anderson, Tatham, y Black, 2005). 
Para la determinación del número de factores, a través del análisis paralelo, se sugirió la extracción de un solo factor. Mediante el método mínimos cuadrados no ponderados (ULS) sin rotación, se obtuvo $40.5 \%$ de la varianza total explicada del instrumento, el cual es un valor suficiente para determinar la uni dimensionalidad de un instrumento (Carmines y Zeller, 1979). En este análisis, las cargas factoriales oscilaron entre .422 y .645, valores considerados adecuados. En la tabla 1 se pueden apreciar las cargas factoriales de los ítems de la versión final del ESIL.

\section{Tabla 1}

Estructura factorial de la Escala de Identidad Local (ESIL)

\begin{tabular}{clc}
\hline & \multicolumn{1}{c}{ Ítem } & $\lambda$ \\
\hline 1 & Me identifico con el patrimonio de mi distrito. & .490 \\
2 & Siento orgullo de que en mi distrito haya valiosos lugares turísticos. & .484 \\
3 & Me llena de orgullo saber que mi distrito es reconocido mundialmente por su & .543 \\
4 & participación y organización. & .466 \\
5 & Me gusta escuchar sobre la historia de fundación de mi distrito. & .488 \\
6 & Me considero parte de las organizaciones de mi distrito. & .482 \\
7 & Me siento parte de la comunidad. & .645 \\
8 & Estoy dispuesto a defender mi distrito ante cualquier tipo de ofensa. & .618 \\
9 & Me gusta promover los logros obtenidos por mi distrito. & .514 \\
10 & Mantengo siempre presente mi disposición de colaborar con el desarrollo de mi & .599 \\
11 & La población del distrito debe sentirse orgullosa de nuestro origen cultural. & .552 \\
12 & Me siento orgulloso/a del pasado histórico de nuestros fundadores locales. & .604 \\
13 & Me siento comprometido/a con mi distrito. & .594 \\
14 & Revalorar nuestras raíces fortalece nuestra identidad local. & .614 \\
15 & Preferiría haber nacido en otro distrito. & .422 \\
\hline
\end{tabular}

Nota: factor de ponderación >.32 (Tabachnick y Fidell, 2001). $\lambda=$ cargas factoriales

La Confiabilidad de la Escala de Identidad Local - ESIL, obtenida a partir del Coeficiente Alfa de Cronbach, presenta un valor de .857 , lo cual es considerado cualitativamente como un valor bueno. Este índice refleja que los ítems de la escala final propuesta son consistentes para discriminar la variable propuesta. Los niveles de Identidad Local quedan establecidos del siguiente modo: Nivel alto con puntajes entre 61 - 75 puntos, Nivel promedio con puntajes entre 51 a 60 puntos y nivel bajo con puntajes entre 30 y 50 puntos.

\section{Análisis psicométrico de la Escala del Ejercicio de la Ciudadanía - EECI (Pacheco, 2010).}

Sobre el Análisis de las propiedades psicométricas de la Escala del Ejercicio de la Ciudadanía - EECI, los resultados de la validez de contenido indican que veinte (20) de los cuarenta (40) ítems originales del EECI presentados a los jueces fueron aceptados como representativos del universo de contenido de la Escala del Ejercicio de la Ciudadanía, pues los coeficientes $\mathrm{V}$ de Aiken fueron significativos a una $\mathrm{p}<.05$, que en el caso de 10 jueces deben ser mayores a .80 (Escurra, 1989), lo que garantiza que la prueba presenta validez de contenido.

Al establecer la validez de constructo de los ítems de la Escala de Ejercicio de la Ciudadanía EECI, a través del criterio de constructo mediante las correlaciones ítem-test, se aprecia en la tabla 2 que 19 ítems de los 20 aceptados por el criterio de jueces presentaron 
correlaciones significativas mayores a .30 .

Análisis Factorial Exploratorio - AFE, primero se exploró si los datos mostraban bondad de ajuste respecto a la matriz de relaciones lineales, condición fundamental para realizar el análisis factorial. Se obtuvo una matriz de correlaciones diferente de cero, Barttlett- $\chi^{2}(\mathrm{gl}: 171)=$ $1199.3, \mathrm{p}<.01$; KMO de 0.87, valor considerado adecuado (Hair, Anderson, Tatham, y Black, 2005).

Para la determinación del número de factores, a través del análisis paralelo, se sugirió la extracción de dos factores, lo cual coincide con el modelo propuesto en la construcción de la escala. Mediante el método mínimos cuadrados no ponderados (ULS) con rotación oblimin directo (debido a que ambos factores obtuvieron un grado de correlación moderada, $r=.39$ ), se obtuvo $41.1 \%$ de la varianza total explicada del instrumento, el cual indicaría la estructura bifactorial del instrumento. En este análisis, las cargas factoriales oscilaron entre .471 y .693, valores considerados adecuados. En la tabla 2 se pueden apreciar las cargas factoriales de los ítems finales del EECI para ambos factores obtenidos. 
Tabla 2

Estructura factorial de la Escala de Ejercicio de la Ciudadanía (EECI)

\begin{tabular}{|c|c|c|c|}
\hline & Ítem & IC & $\mathrm{CM}$ \\
\hline 1 & $\begin{array}{l}\text { Me interesa que la participación ciudadana se } \\
\text { promueva desde la escuela }\end{array}$ & .693 & .096 \\
\hline 2 & $\begin{array}{l}\text { Me interesaría participar en actividades para } \\
\text { proteger el medio ambiente }\end{array}$ & .649 & .141 \\
\hline 3 & $\begin{array}{l}\text { Tengo la intención de ejercer una } \\
\text { responsabilidad social con mi país }\end{array}$ & .594 & .384 \\
\hline 4 & Tengo toda la intención de obedecer las leyes & .578 & .200 \\
\hline 5 & $\begin{array}{l}\text { Tengo la intención de participar en actividades } \\
\text { de defensa de los derechos humanos }\end{array}$ & .576 & .249 \\
\hline 6 & $\begin{array}{l}\text { Tengo la intención de informarme para dar un } \\
\text { voto consciente cuando participe de las elecciones }\end{array}$ & .573 & .260 \\
\hline 7 & $\begin{array}{l}\text { Tengo la intención de ser parte de los espacios de } \\
\text { participación en mi comunidad }\end{array}$ & .566 & .363 \\
\hline 8 & $\begin{array}{l}\text { Tengo la intensión de informarme sobre los } \\
\text { espacios de participación en mi comunidad }\end{array}$ & .563 & .322 \\
\hline 9 & $\begin{array}{l}\text { Tengo la intención de realizar acciones para } \\
\text { resolver los problemas que hay en mi comunidad }\end{array}$ & .542 & .392 \\
\hline 10 & $\begin{array}{l}\text { Tengo la intención de ejercer mis derechos } \\
\text { participando en mi comunidad }\end{array}$ & .471 & .340 \\
\hline 11 & $\begin{array}{l}\text { Invierto mi tiempo en apoyar iniciativas de } \\
\text { cambio en mi comunidad }\end{array}$ & .266 & .613 \\
\hline 12 & $\begin{array}{l}\text { Colaboro con otras personas para hacer de mi } \\
\text { comunidad un mejor lugar }\end{array}$ & .364 & .595 \\
\hline 13 & $\begin{array}{l}\text { He dado mis propuestas al menos en un espacio } \\
\text { de participación. }\end{array}$ & .267 & .589 \\
\hline 14 & $\begin{array}{l}\text { He participado de iniciativas para solucionar un } \\
\text { problema de mi comunidad }\end{array}$ & .281 & .574 \\
\hline 15 & $\begin{array}{l}\text { Continuamente participo en actividades que } \\
\text { contribuyen al bien común }\end{array}$ & .226 & .572 \\
\hline 16 & $\begin{array}{l}\text { Hasta el momento he participado al menos de } \\
\text { una organización social }\end{array}$ & .091 & .552 \\
\hline 17 & $\begin{array}{l}\text { Considero que realizo acciones como agente de } \\
\text { cambio en mi comunidad }\end{array}$ & .339 & .543 \\
\hline 18 & $\begin{array}{l}\text { He realizado un voto informado en las elecciones } \\
\text { en donde he participado }\end{array}$ & .195 & .527 \\
\hline 19 & $\begin{array}{l}\text { He participado en actividades de integración en } \\
\text { la comunidad }\end{array}$ & .225 & .513 \\
\hline
\end{tabular}

Nota: factor de ponderación $>.32$ (Tabachnick y Fidell, 2001). $\mathrm{IC}=$ Intención de la conducta. $\mathrm{CM}=$ Conducta manifiesta. $\lambda=$ cargas factoriales

La Confiabilidad de las dimensiones Escala de Ejercicio de la Ciudadanía - ESIL, obtenida a partir del Coeficiente Alfa de Cronbach, presenta un valor de .83 para el factor de intención de la conducta, y de .80 para conducta manifiesta, lo cual es considerado cualitativamente como un valor bueno. Así mismo se calculó el coeficiente alfa de Cronbach para el puntaje total, obteniéndose un valor también bueno de .85; este índice refleja que los ítems de la escala final propuesta son consistentes para discriminar la variable propuesta. Los niveles del Ejercicio de la Ciudadanía quedan establecidos del siguiente modo: Nivel alto con puntajes entre 75- 95 puntos, Nivel promedio con puntajes entre 63 a 74 puntos y nivel bajo con puntajes entre 30 y 62 puntos. 


\section{Relación entre nivel de identidad local y el ejercicio de la ciudadanía en jóvenes de Villa El Salvador}

La identidad local en los jóvenes de Villa El Salvador, presentó un nivel promedio, como lo demuestra la media del puntaje 55.24. El ejercicio de la ciudadanía en los jóvenes de Villa El Salvador, presentó un nivel promedio, como lo demuestra el la media del puntaje 68,23. La relación entre nivel de identidad local y el ejercicio de la ciudadanía en jóvenes de Villa El Salvador, presentó que el coeficiente de correlación producto momento de Pearson tiene una relación positiva estadísticamente significativa y alta entre el nivel de identidad local y el ejercicio de la ciudadanía $(\mathrm{r}=0.653, \mathrm{p}<0.01)$, de igual manera que con la dimensión intención de la conducta. Esto quiere decir que cuanto mayor es el nivel de identidad local mayor es el nivel del ejercicio de la ciudadanía. Por último, existe una relación estadísticamente significativa y moderada entre la identidad local y la dimensión de conducta manifiesta del ejercicio de la ciudadanía (ver tabla 3).

\section{Tabla 3}

Coeficiente de correlación entre identidad local y el ejercicio de la ciudadanía en los jóvenes de Villa El Salvador.

\begin{tabular}{ccccc}
\hline & IC & CM & \multicolumn{2}{c}{$\begin{array}{c}\text { Ejercicio de la } \\
\text { Ciudadanía }\end{array}$} \\
\cline { 2 - 4 } & $R$ & $r$ & $r$ \\
\hline $\begin{array}{c}\text { Identidad } \\
\text { local }\end{array}$ & $.633^{* *}$ & $.469^{* *}$ & $.653^{* *}$
\end{tabular}

Nota: $r=$ coeficiente de correlación producto momento de Pearson. $\mathrm{IC}=$ Intención de la conducta. $\mathrm{CM}=\mathrm{Conducta}$ manifiesta.

\section{Discusiones}

Los principales hallazgos de la investigación fueron respecto a los instrumentos empleados en el presente estudio, la escala de Identidad Local (ESIL) y la escala del Ejercicio de la ciudadanía (EECI) son instrumentos que han demostrado tener adecuadas propiedades psicométricas para la muestra de jóvenes de Villa El Salvador, por lo que su aplicación en este contexto es pertinente para la obtención de datos válidos y confiables que permitan realizar el estudio y relación de las variables identidad local y ejercicio de la ciudadanía.

Por otro lado, el objetivo principal del estudio fue establecer la relación entre la Identidad local y el Ejercicio de la Ciudadanía, encontrándose una correlación positiva, alta y estadísticamente significativa. Esto quiere decir que cuanto mayor es el nivel de identidad local mayor es el nivel del ejercicio de la ciudadanía en la muestra de jóvenes evaluados.

En la muestra se estudia la manifestación de conducta ciudadanas como participación activa política, actividades de integración local, solución de problemáticas comunes y generación de espacios de dialogo comunal, van a reforzar la formación de la identificación de la persona con su localidad, haciéndolo socialmente más responsable con la problemática del distrito y sintiéndose realmente comprometido y parte de él. Ya que es claro que el ejercer la ciudadanía no solo se refiere al ejercicio de los derechos humanos, sino al ejercicio general de la racionalidad para la búsqueda de solución de problema, vinculados con espacios públicos; es por 
ello que implica ir más allá de solucionar problemáticas de orden personal sino más bien de orden público (Grubits y Vera, 2005), lo cual tiene más que ver con una identificación y compromiso con la localidad o comunidad en la que la persona se desenvuelve.

Otro punto importante hallado es que, si bien es cierto que se halló una relación fuerte entre identidad local y ejercicio de la ciudadanía, ésta fue más fuerte con la dimensión intención de la conducta que con la de conducta manifiesta, la cual fue moderada. Lo cual nos lleva a pensar en que si bien es cierto hay una adecuada identificación y compromiso con el distrito y que existe intereses y deseos con respecto a tener una participación más activa en la comunidad, la realización de conducta no se da en un grado alto, por lo que es necesario generar aún más espacios de participación ciudadana y fomentar actividades de dialogo e integración para la solución de las problemáticas del distrito.

En cuanto a la relación de ambas variables según la edad se encontraron correlaciones estadísticamente significativas y entre moderadas y altas, para los grupos de 14 a 18 años y de 19 a 24 años. Aunque en el segundo grupo los coeficientes tienden a ser mayores, con respecto a los más jóvenes. Esto permite deducir que en la muestra de estudio los participantes más jóvenes con una adecuada identidad local y que se sienten identificados con su comunidad pueden manifestar conductas de ciudadanía que permitan su participación en su contexto social, sin embargo ésta es más fuerte conforme tienen más edad, ello debido probablemente a un mayor compromiso e interés con su realidad y sumado a que la construcción de su identidad local va consolidándose más conforme tienen más participación con las actividades políticas y de integración que se realizan.

En cuanto al grado académico, se observan correlaciones estadísticamente significativas entre las variables de estudio. Lo cual indica que en los jóvenes con un nivel de formación de educación secundaria o superior se puede dar un adecuado ejercicio de la ciudadanía en su comunidad con un alto grado de identificación local. Esta asociación es más fuerte en la dimensión de conducta manifiesta en los jóvenes con un nivel secundario en comparación a los jóvenes universitarios. Esto implica que los estudiantes universitarios tienen menor espacio de participación dentro de su comunidad, debido quizá a la demanda de tiempo y esfuerzo que implica el acceder a estudios superiores, lo cual puede disminuir el espacio de participación activa que puedan asumir dentro de su localidad.

Por último, relacionado con el sexo tenemos que en ambos casos existe una asociación alta y estadísticamente significativa, aunque es algo mayor en las mujeres. Si bien al respecto como hallaron Inhoff, et al. (2011) son los varones quienes presentan un mayor interés por el debate político y eficacia política local, pues está vinculado con las diferencias sociales establecidas por cada género, las cuales normalmente vinculan y propician un mayor involucramiento y protagonismo en el ámbito de la participación ciudadana. Sin embargo, para la presente muestra han sido las mujeres quienes han presentado una asociación mayor con respecto a la identidad local y el ejercicio de la ciudadanía. Podemos atribuir a que se viene promoviendo espacios de participación donde las mujeres vienen participando de forma activa, como las asociaciones juveniles y municipios escolares, que tienen estatutos que sugieren cuotas de participación de mujeres, estas prácticas vienen fortaleciendo la equidad de género, y en forma 
recíproca las mujeres vienen empoderándose como una forma de autoafirmarse como agentes de cambio (Pacheco, 2010).

De acuerdo a los hallazgos encontrados, consideramos que el presente estudio resulta un aporte significativo en primer lugar en la medida que se confirma la consistencia de los instrumentos empleados en poblaciones de jóvenes. Asimismo, dado que en nuestro contexto temas relacionados al campo de la psicología social y dentro de ella la identidad local y el ejercicio de la ciudadanía siguen siendo poco desarrollados, pensamos que la presente investigación abre nuevas posibilidades de mantener una mirada en la investigación de fenómenos sociales en los jóvenes.

\section{Conclusiones}

Los ítems del ESIL como del EECI obtuvieron adecuados coeficientes V de Aiken, lo cual es un indicador de que presentan validez de contenido.

En cuanto a la validez de constructo, tanto para el ESIL como el EECI se obtuvieron adecuadas correlaciones ítem-test, lo que garantiza que los ítems de las versiones finales de ambas pruebas presentan las condiciones métricas necesarias para su inclusión en las escalas elaboradas.

El ESIL presenta una estructura factorial unidimensional, lo cual genera un solo puntaje interpretable. El EECI presenta una estructura bifactorial, con dos factores interpretables: intención de la conducta y conducta manifiesta.

En cuanto a la confiabilidad por consistencia interna se han obtenido buenos coeficientes alfa de Cronbach para ambas escalas.

Existe una correlación, estadísticamente significativa, positiva y alta entre las variables identidad local y ejercicio de la ciudadanía, así como también con la dimensión intención de la conducta. Con la dimensión conducta manifiesta existe una correlación positiva y moderada.

Existe una correlación estadísticamente significativa entre las variables identidad local y ejercicio de la ciudadanía con sus dos dimensiones, tanto en varones como en mujeres, siendo mayor en las mujeres.

Existe una correlación alta y estadísticamente significativa entre identidad local y ejercicio de la ciudadanía con sus dos dimensiones, según el grado académico obtenido.

Existe una correlación alta y estadísticamente significativa entre identidad local y ejercicio de la ciudadanía con sus dos dimensiones, según en las edades entre 14 y 18 años, y 19 a 24 años. 


\section{Bibliografía}

Capriotti, P. (1992). Imagen de empresa. Estrategia para una comunicación integrada. Barcelona: El Ateneo S.A.

Carmines, E. y Zeller, R. (1979). Reliability and validity assessment. California: Sage

Castells, M. (1997). La Era de la Información. Vol II: El Poder de la Identidad. México: Siglo XXI.

Crouch, C. (1999). La ampliación de la ciudadanía social y económica y la participación. Madrid: Siglo XXI.

Cruz, B., \& Pedraza, J. (2011). Cultura e identidad regional y sus implicaciones en el ámbito regional y municipal. México: IAPEM.

De Zan, J. (2008). Memoria e Identidad. Tópicos. Revista de Filosofía de Santa Fe, 16, 41-67. Recuperado de http://www.scielo.org.ar/pdf/topicos/n16/n16a03.pdf

Donati, P. (1999). La ciudadanía societaria. Granada: Universidad de Granada.

Dromi, R. (2007). Ciudad y municipio. Gerenciamiento y gobernabilidad local. Madrid: Hispania Libros.

Escurra, L. (1989) Cuantificación de la Validez de Contenido por Criterio de Jueces. Revista de Psicología - PUCP, 6, 103-111. Recuperado de https://bit.ly/2CGfZOB

Gadea, E. (2007). Las políticas de participación ciudadana: Nuevas formas de relación entre la administración pública y la ciudadanía. El caso de la ciudad de Valencia y su área metropolitana (Tesis doctoral). Universidad de Valencia, Madrid-España.

García Canclini, N. (1999). Los usos sociales del patrimonio cultural. En E. Aguilar Criado (Ed.), Patrimonio etnológico. Nuevas perspectivas de estudios (pp. 16-33). España: Instituto Andaluz del Patrimonio Histórico.

Geertz, C. (2000). La interpretación de las culturas. Barcelona: Gedisa.

Giménez, G. (2007). Estudios sobre la Cultura y las Identidades Sociales. México: CONACULTA.

Grubits, S. y Vera, J. A. (2005). Construcción de la identidad y la ciudadanía. Ra Ximhai, 1(3), 471-488.

Hair, J., Anderson, R., Tatham, R. \& Black, W. (1999). Análisis Multivariante. Madrid: Prentice Hall.

Heller, A. (2002). Sociología de la Vida Cotidiana. Barcelona: Península.

Hernández, R., Fernández, C., \& Baptista, P. (2006). Metodología de la investigación. (4ª ed.). México: McGraw-Hill.

INEI (2008). Censos Nacionales 2007: XI de Población y VI de Vivienda. Perú: Crecimiento y distribución de la población, 2007. Recuperado de https://bit.ly/2gvNsBG

Imhoff, D., Gutiérrez, Y. y Brussino, S. (2011). Jóvenes y cuidadanía: indagación acerca del modo de ejercicio de la ciudadanía de los/as jóvenes a partir de la relación de las orientaciones psicológicas de la politización juvenil con la participación política. Revista Tesis, 1, 21-38.

Kymlicka, W., \& Norman, W. (1997). El retorno del ciudadano. Una revisión de la producción reciente en teoría de la ciudadanía. México: La Política.

Montero, I. \& León, O. (2002). Clasificación y descripción de las metodologías de investigación en Psicología. International Journal of Clinical and Health Psychology, 2(3), 503-508.

Morán, Mª L., \& Benedicto, J. (2000). Jóvenes y ciudadanos. Propuestas para el análisis de las culturas ciudadanas de la juventud. Madrid: Instituto de la Juventud.

Esta obra se comparte bajo la licencia Creative Common Atribución-No Comercial 4.0 International (CC BY-NC 4.0) 
Morin, E. (2002). La cabeza bien puesta. Repensar la reforma. Reformar el pensamiento. Buenos Aires: Nueva Visión.

Municipalidad de Villa El Salvador. (2006). Plan Integral de Desarrollo Concertado de Villa El Salvador al 2021 (PIDCVES 2021). Recuperado de https://bit.ly/2S1dRqt

Nidding, M. (2001). Turismo sostenible, comunidad local y competencias para el desarrollo. En A. Lebian (Ed.), Turismo cultural y desarrollo sostenible (pp. 101-128). Murcia, España: Universidad de Murcia.

Pacheco, D. (2010). Elaboración de una Escala de Identidad Local y de una escala del ejercicio de la ciudadanía. Manuscrito inédito.

Pacheco, D. (2016). Identidad Local y Ejercicio de la Ciudadanía en Jóvenes de Villa El Salvador (Licenciatura). Universidad Nacional Federico Villarreal.

Passerin D’Entrèves, M. (1992). Hanna Arendt and the idea of citizenship. En C. Mouffé (Ed.), Dimensions of Radical Democracy (pp. 145-168). London: Verso.

Pérez Ledesma, M. (2000): Ciudadanía y Democracia. Madrid: Pablo Iglesias.

Portocarrero, C. (1998). Elaboración de una Escala de Identidad Nacional. Manuscrito inédito.

Real Academia Española. (2013). Diccionario de la Lengua Española. (25 ed.). Madrid: Espasa Calpe.

Rizo, M. (2005). Me comunico, luego existo. El Papel de la Comunicación en la construcción de Identidades. Culturales, 1(1),124-142. Recuperado de https://bit.ly/2LNbd6N

Rostworoski, M. (1987). Somos un país acomplejado. El zorro de abajo. 7 Junio 44-49.

Sánchez, H., \& Reyes, C. (2009). Metodología y diseño en la investigación científica. Lima. Editorial Universitaria URP.

Sermeño, A. (2004). Ciudadanía y teoría democrática. Metapolítica, 8(33), 87-94.

Sojo, C. (2002). La noción de ciudadanía en el debate latinoamericano. Revista de la CEPAL, 76, 25-38. Recuperado de https://bit.ly/2xAx9tp

Tabachnick, B., \& Fidell, L. (2001). Using multivariate statistics (5ta.ed.). Nueva York: Harper \& Row.

Turner, B. S. (1993). Contemporary Problems in the Theory of Citizenship. London: Sage.

Zúñiga, C., \& Asún, R. (2003). Identidad Regional en contexto de cambio. Un estudio en la Araucanía, Chile. Psicología Política, 26, 73-92. Recuperado de https://bit.ly/2ESZ6SF 